\title{
Tuberous Sclerosis Complex: Rare Disease with Significant Social Impact (Case Series)
}

\author{
J. Breza jr. (Jan Breza jr.)', B. Novotna (Barbora Novotna)²
}

Department of Urology, University Hospital Bratislava, SK

Original Article

${ }^{2}$ Clinic and Polyclinic for Pediatrics, Dresden University of Technology, DE

\section{E-mail address:}

janbreza@gmail.com

\section{Reprint address:}

Jan Breza

Department of Urology, University Hospital Bratislava

Limbova 5

83305 Bratislava

SK

Suource: Clinical Social Work and Health Intervention

Volume: 8

Issue: 4

Pages: $17-24$

Cited references: 12

\section{Reviewers:}

Vlastimil Kozon

Allgemeines Krankenhaus - Medizinischer Universitätscampus Vienna, AU

John Mutuku

MIC Nairobi, KE

\section{Key words:}

Tuberous Sclerosis Complex. Gene Mutations. Epilepsy. Mental Impairment. Angiomyolipomas. Hamartomas.

\section{Publisher:}

International Society of Applied Preventive Medicine i-gap

CSWHI 2017; 8(4): 17 - 24; DOI 10.22359/cswhi_8_4_02 @ 2017 Clinical Social Work and Health Intervention

\section{Abstract:}

The disease called the tuberous sclerosis complex has, besides its medical impact, also a significant social impact. It negatively affects the quality of life not only of the affected patients but also of their families, as this disease also occurs in a familial form. The social aspects of this disease are also reflected in the need to create specific conditions for 
the upbringing, education and care of the patients suffering from the tuberous sclerosis complex.

In clinical practice we only rarely come upon patients affected by the tuberous sclerosis complex. Therefore, the experience in the diagnostics and management of these patients is quite limited. Diagnostics of the tuberous sclerosis complex is more difficult due to the fact that subjective symptoms and objective signs are not specific for the tuberous sclerosis complex, but can be the signs of other diseases.

Tuberous sclerosis complex is an autosomal dominant hereditary disease that is the result of mutations of two tumor suppressor genes and is characterized by the formation of benign tumors, hamartomas, in several organ systems. In about $80 \%$ of cases the mutations are acquired, the cause of mutation is unknown. Autosomal dominant gene mutations are the cause of about $20 \%$ of cases of tuberous sclerosis complex, and affliction of more family members is found. Most commonly, in patients with tuberous sclerosis, neurological and psychiatric symptoms are present in the central nervous system. Renal hamartomas are called angiomyolipomas. After neurological and skin changes, renal angiomyolipomas are ranked third in the incidence rate in patients with tuberous sclerosis.

The authors analyze their own clinical experience with the diagnostics and treatment of children and adult patients with the tuberous sclerosis complex. The study includes 14 children ( 8 girls and 6 boys aged 7 to 15 years) and 12 adult patients ( 9 women and 3 males aged 17 to 42 years). The girls and women predominated (66.7\%) over male patients $(33.3 \%)$.

All patients were polysymptomatic, symptomatology was highly complex and polymorphic. In every patient the signs of simultaneous affliction of at least two organ systems were found. There was no common symptom to all patients, and no symptom was absolutely pathognomonic for the tuberous sclerosis complex. Neurological symptomatology was the dominant finding in children. Very significant is the recognition that affliction of the brain resulted in almost all children, besides topical symptomatology, also in epilepsy, intellectual impairment disorders in the form of mental retardation of various degrees and autism.

In adult patients, affliction of the kidneys by angiomyolipomas was the predominant form of the tuberous sclerosis complex. In most of them simultaneous signs of affected skin and nails was observed.

Since virtually in all cases of impairment of the brain, kidneys and also of the heart, the disease is life-threatening and surgically incurable, it is reasonable to consider the alternative treatment by administration of an inhibitor of the mTOR signaling pathway.

\section{Conflict of interests:}

None. 


\section{Introduction}

The complex of tuberous sclerosis is an autosomal dominant hereditary neuro-cutaneous disease characterized by pleomorphic features and affecting several organ systems. It is manifested by the formation of benign tumors, hamartomas, in the brain, kidneys, heart, liver, eyes, mouth and on the skin.

The incidence of the tuberous sclerosis complex is $1: 6,000$ to $1: 10,000$ of births. The population prevalence is estimated at 1:20,000. Globally, about one and half million patients are affected by tuberous sclerosis (Osborne et al. 1991, O'Callaghan et al. 1998, Northrup and Krueger, 2013).

Two gene mutations, TSC1 on chromosome $9(9 \mathrm{q} 34)$ and TSC2 on chromosome 16 (16p13) are responsible for tuberous sclerosis development. TSC1 and TSC2 belong to tumor suppressor genes that play an important role in inhibiting the mTOR signaling pathway responsible for cell proliferation (Jozwiak et al., 2005, Au et al., 2007). These genes encode the information responsible for the synthesis of two proteins - hamartin and tuberin - which together form an intracellular complex blocking excessive cell proliferation and differentiation. If a mutation of one of the genes results in a loss of the blockade, the affected cell are propagated uncontrollably and tumors develop. Since hamartin and tuberin are formed in most tissues, the complex of tuberous sclerosis can be manifested by the formation of tumors in several organs (Crino, 2013). Typical manifestation of tuberous sclerosis complex includes tumors, hamartomas in the brain and in the kidneys. Since clinical experience with patients suffering from these tumors within the tuberous sclerosis complex is rare, the knowledge on the diagnostics and management of these patients is quite limited (Novotna, 2013).

\section{Patients}

The study included 14 children ( 8 girls and 6 boys aged 7 to 15 years) and 12 adult patients ( 9 women and 3 men aged 17 to 42 years) who suffered from various symptoms of tuberous sclerosis. All of these patients complied with the main or secondary criteria formulated by the National Association of Tuberous Sclerosis in 2012 and published in year 2013. The female patients predominated $(66.7 \%)$ over male patients $(33.3 \%)$.

All patients underwent a complete clinical evaluation. All the subjective symptoms were taken into account, similarly the objective features and circumstances in which the tuberous sclerosis complex was diagnosed. Emphasis was put on the family history of the tuberous sclerosis complex in genetically related family members.

The mental condition of the patients and the level of intelligence in children were assessed using special questionnaires and standardized psychological examination. Based on IQ, the patients were categorized into three categories, from mental retardation with IQ of less than 70 to the age-appropriate intelligence with IQ of more than 85 .

Imaging and functional examinations of individual organ systems followed with particular emphasis on the brain and the kidneys visualized by CT and/or MRI examinations.

\section{Results}

The familial incidence was found in $18.1 \%$ of cases. The category of cases of $f a-$ milial tuberous sclerosis complex includes 7 and 9 year old girls, cousins who inherited mutation of TSC2 gene from their fathers and from the grandmother. The third case was an 11 year old girl whose father had exhibited a symptomatology of the $t u$ berous sclerosis complex at the mutation of the TSC2 gene. Another patient, 42 year old woman has a 13-year-old son who suffers 
from the same difficulties as his mother but this patient has not been examined and included in the study.

Symptomatology of the tuberous sclerosis complex in children was complex and markedly polymorphic. Symptomatology of the central nervous system disorder characterized by epilepsy and mental retardation was dominant. This affects 7 out of 8 girls and 5 out of 6 boys (Table 1). all cases was in the structural changes in the brain. In all girls and in 5 out of the 6 boys, numerous subependymal nodules, subcortical and cortical tubers, and lesions of the white matter of the brain were proven.

The second most common symptom of tuberous sclerosis in children was heart affliction. Hamartomas in the heart were found echocardiographically in 3 out of 8 girls $(37.5 \%)$ and in all 6 boys. In all cases,

Table 1: Affliction of organ systems in children with tuberous sclerosis complex.

\begin{tabular}{|l|c|c|}
\hline & Girls $n=8$ & Boys $\mathrm{n}=6$ \\
\hline Affliction of the brain & $7 / 8 \quad 87.5 \%$ & $5 / 6 \quad 83.3 \%$ \\
\hline Affliction of the heart & $3 / 837.5 \%$ & $6 / 6 \quad 100 \%$ \\
\hline Affliction of the kidneys & $4 / 8 \quad 50 \%$ & $4 / 6 \quad 66.7 \%$ \\
\hline Affliction of the skin, including the nails & $6 / 875 \%$ & $4 / 6 \quad 66.7 \%$ \\
\hline
\end{tabular}

Epilepsy and mental retardation in tuberous sclerosis complex diagnosis are not among the main or secondary diagnostic criteria. However, the incidence of epilepsy and mental retardation was surprisingly high in patients diagnosed with tuberous sclerosis (Table 2). these were one or more hamartomas (1 to 6) of low hemodynamic severity. In adult patients, heart affliction was not observed. Multiple renal angiomyolipomas occurred in $50 \%$ of girls and $67 \%$ of boys. Angiomyolipomas in children were mostly small, with a diameter of up to $8 \mathrm{~mm}$, greater in

Table 2: IQ values in patients with tuberous sclerosis complex.

\begin{tabular}{|l|c|c|}
\hline IQ & Girls $n=7$ & Boys $n=5$ \\
\hline less than 70 & $4 / 757.1 \%$ & $4 / 580 \%$ \\
\hline $70-85$ & $1 / 714.3 \%$ & - \\
\hline more than 85 & $2 / 728.6 \%$ & $1 / 520 \%$ \\
\hline
\end{tabular}

Epilepsy occurred in all patients with mental retardation. Epilepsy, however, occurred also in one girl and one boy of age-adequate intelligence. The cause of epilepsy in only two children. Angiomyolipomas were asymptomatic in all children.

In the group of 12 adult patients, renal involvement was the dominant sign (Table 3). 
Table 3: Impairment of organ systems with tuberous sclerosis complex in adult patients.

\begin{tabular}{|l|r|r|}
\hline & Women $\mathrm{n}=9$ & Men $\mathrm{n}=3$ \\
\hline Affliction of the kidneys & $9 / 9 \quad 100 \%$ & $2 / 3 \quad 66.7 \%$ \\
\hline Affliction of the brain & $2 / 922.2 \%$ & $2 / 3 \quad 66.7 \%$ \\
\hline Affliction of the skin and nails & $8 / 9 \quad 88.9 \%$ & $1 / 3 \quad 33.3 \%$ \\
\hline Affliction of the lungs (LAM) & $2 / 922.2 \%$ & \multicolumn{1}{|c|}{-} \\
\hline
\end{tabular}

Renal angiomyolipomas were demonstrated in all adult patients with tuberous sclerosis complex. In all cases, multiple tumors were presented; in 10 patients angiomyolipomas occurred on both sides. All patients complained of intermittent blunt pain in the lumbar region. In three women, angiomyolipomas manifested dramatically through so-called Wunderlich syndrome, hemorrhagic shock due to massive retroperitoneal bleeding from the ruptured large angiomyolipoma of the kidney.

In two female patients we diagnosed an incipient stage of chronic renal insufficien$c y$ due to destruction of the renal parenchyma by angiomyolipomas.

Especially from an aesthetic point of view, 8 of 9 women and 2 adolescent girls were very uncomfortable with the lesions of the skin of the face and nails. The affected patients had extensive changes in the face due to numerous perioral and perinasal angiofibromas. In two female patients, the disease was manifested also by paraungual fibromas on the fingers of hands and feet.

Genetic tests were performed in 19 patients (Table 4)
Patients with the TSC2 gene mutation had more severe clinical manifestations of the disease with a higher incidence of epilepsy, more severe kidney impairment and more pronounced facial angiofibromas.

In five adult women, diagnosis of the tuberous sclerosis complex was determined without genetic examination only on the basis of clinical examination, meeting the baseline criteria recommended by the International TSC Consensus Conference in 2012. In three adult patients, genetic examination was performed because of family counseling, the current testing methods, however, failed to prove any mutation in the TSC1 or TSC2 gene.

\section{Discussion}

The complex of tuberous sclerosis is a disease very variable in its manifestations. The expression of the specific genotype are different phenotypic manifestations of the disease manifesting in different ages of the patients at the onset of the disease, different severity of the disease and various subjective symptoms

Table 4: Mutations of the TSC2 gene in patients with tuberous sclerosis complex.

\begin{tabular}{|l|c|c|c|}
\hline & Girls $\mathrm{n}=8$ & Boys $\mathrm{n}=6$ & Adults $\mathrm{n}=10$ \\
\hline no changes & $1 / 8$ & $1 / 6$ & $3 / 10$ \\
\hline mutation de novo & $4 / 8$ & $5 / 6$ & $2 / 10$ \\
\hline congenital mutation & $3 / 8$ & - & - \\
\hline
\end{tabular}


as well as objective signs. In addition, patients with tuberous sclerosis complex have a higher risk of developing malignancies. All these aspects have to be taken into consideration when planning treatment (Rakowski et al. 2006).

In most cases, the treatment of the tuberous sclerosis complex is only palliative, depending on the extent of affliction of single organ systems.

More than $80 \%$ of patients with tuberous sclerosis experience changes in their kidneys during their lifetime. The first symptoms indicative of renal involvement are most often manifested in the second or third decade of life. However, we have observed also young children in whom kidneys have been afflicted by angiomyolipomas. Renal involvement is the second most common cause of death of patients with tuberous sclerosis complex (Shepherd et al., 1991). The most common form of renal involvement in tuberous sclerosis patients is angiomyolipoma. These are the tumors formed by abnormal thick-walled vessels, smooth muscle fibers and fat tissue. They are mostly found on the kidney surface, they are small (a few centimeters in diameter), usually grow slowly, but they can get bigger. Up to $70-90 \%$ of patients with tuberous sclerosis have multiple angiomyolipomas. They occur on both sides, and usually grow slowly. Tumor growth or an increased number of tumors occur in $60 \%$ of patients. The growth often results in an aneurysm of the arterial blood vessels of angiomyolipomas and in a formation of so-called pirated blood vessel bed. The growth appears to depend on the hormonal status of the affected patients, as evidenced by the prevalence of these tumors in women and virtually zero in prepubertal patients.

Diagnostics of tuberous sclerosis complex is based on targeted history; on the basis of clinical criteria; and/or the results of genetic testing (Rose et al., 1991). Genetic testing is not required to establish the diagnosis in patients who meet the clinical criteria for a definitive diagnosis. According to the Consensus Guidelines for Diagnosis of TSC, renal angiomyolipomas are among the major diagnostic criteria for tuberous sclerosis complex. In addition to numerous and bilateral renal angiomyolipomas, in our patients we also found other major diagnostic criteria face angiofibromas, ungual fibromas, and lymphangioleiomyomatosis of the lung.

Kidney involvement with angiomyolipomas was the dominant symptom of the tuberous sclerosis complex in our group of patients. Women are affected by renal angiomyolipomas more often than men. In the studied patients, we observed both subjective symptoms as well as objective signs of renal disease. Clinical manifestations of renal angiomyolipomas include anorexia, pain, palpitations, hematuria, hypertension, anemia. In three female patients, we urgently addressed a life-threatening situation, hemorrhagic shock from massive bleeding to the retroperitoneum due to a rupture of dysplastic aneurysmal vascular vessels in the tumor. The risk of rupture is particularly threatening to patients with large angiomyolipomas (diameter greater than $4-6 \mathrm{~cm}$ ) and aneurysmal vessels with a diameter of more than $5 \mathrm{~mm}$ (Breza et al., 2010).

The number and size of angiomyolipomas as well as their relationship to renal parenchyma and the renal excretory system can be monitored by ultrasonography of the kidneys, and/or by $\mathrm{CT}$ and MR examinations. CT and MR examinations allow obtaining information not only about the kidneys, but also about other abdominal or retroperitoneal organs, on the vascular supply of the kidneys and angiomyolipomas and on 
the nature and extent of their pathological disability.

In some patients with tuberous sclerosis complex, most commonly in women after the menarche, pulmonary lymphangioleiomyomatosis can occur with benign metastases of smooth muscle cells of angiomyolipomas. The most accurate diagnostics of this complication is achieved by the so-called high resolution lung $\mathrm{CT}$. The treatment is a palliative use of everolimus. Terminal stages of respiratory insufficiency include lung transplantation (Hancock and Osborne, 2002, Northrup and Krueger, 2013).

The treatment of renal angiomyolipomas must maintain as much functional parenchyma as possible. In asymptomatic patients the procedure is conservative, the patients are followed by the described biochemical and visualization methods. Preventive surgical treatment is considered for rapidly growing angiomyolipomas and large angiomyolipomas (with a diameter of 5 or more $\mathrm{cm}$ ) with a risk of tumor rupture. Preference is given to nephron sparing surgery in which only the pathological focus is removed from the kidney but the kidney is retained. In the case of bleeding angiomyolipomas, targeted selective embolization may be used as the first treatment step. A ruptured angiomyolipoma with severe retroperitoneal hemorrhage and hemorrhagic shock is indicative of urgent surgical revision, usually ending with nephrectomy of the affected kidney. In exceptional cases, also in such a situation a kidney saving operation can be performed.

Causal treatment with the mTOR inhibitor is preferred in patients with growing but still asymptomatic multiple angiomyolipomas (Novotna 2013).

In case of chronic renal insufficiency caused by destruction of renal parenchyma by angiomyolipomas the possibility of kidney transplantation from a dead donor may be considered in appropriate patients with tuberous sclerosis complex. Everolimus, the mTOR signaling pathway inhibitor, becomes part of basal immunosuppression in these patients.

\section{Conclusion}

In clinical practice, Urologists only rarely meet patients suffering from the tuberous sclerosis complex. Therefore, experience with the management of this disease is very limited in affected patients. Diagnostics of tuberous sclerosis complex is all the more demanding because the patients are usually poly-symptomatic, subjective but also objective symptoms are not specific to the complex of tuberous sclerosis but can also be the signs of other diseases. In all patients, we found the signs of simultaneous involvement of at least two organ systems. There were no symptom common to all patients, and no symptom was absolutely pathognomonic for the tuberous sclerosis complex.

Patients with kidney disease affected by tuberous sclerosis complex should be monitored on a long-term and systematic basis, in a timely and robust manner, while at the same time complications resulting from kidney angiomyolipomas must be resolved sparingly.

The complex of tuberous sclerosis is a disease that cannot be prevented. However, based on knowledge and experience, it is possible to modify the course of the disease, improve the quality of life and extend the survival of some patients.

\section{References}

1. AU KS, WILLIAMS AT, ROACH ES, BATCHELOR L, SPARAGANA SP, DELGADO MR, WHELESS JW, BAUMGARTNER JE, ROA BB, WILSON CM, SMITH-KNUPPEL TK, CHEUNG MY, WHITTEMORE VH, KING TM, NORTHRUP H (2007) Genotype/phenotype correlation in 325 individuals referred for 
a diagnosis of tuberous sclerosis complex in the United States. Genet. Med.;9:88-100.

2. BREZA J JR, BARDOS A, BREZA J ST (2010) Wunderlich Syndrome: Rare complication angiomyolipoma of the kidney. Medical Horizon, 59:502-506.

3. CRINO PB (2013) Evolving neurobiology of tuberous sclerosis complex. Acta Neuropathologica; 125:317-332.

4. CURATOLO P, BOMBARDIERI R, JOZWIAK S (2002) Tuberous sclerosis. The Lancet 2008;372:657-668.

5. HANCOCK E, OSBORNE J (2002) Lymphangioleiomyomatosis: a review of the literature. Respir. Med.;96:1-6.

6. JOZWIAK J, JOZWIAK S, GRZELA T, LAZARCZYK M (2005) Positive and negative regulation of TSC2 activity and its effect on downstream effectors of the mTOR pathway. Neuromolecular. Med.; 7:287296.

7. NORTHRUP H, KRUEGER D (2013) On the behalf of the International Tuberous Sclerosis Complex Consensus Group: Tuberous Sclerosis Complex Diagnostic Criteria Update: Recommendation of
2012 International Tuberous Sclerosis Complex Consensus Group. Pediatr. Neurol.;49:243-254.

8. NOVOTNA B (2013) The complex of tubercular sclerosis. Slovak Pedagogical Publishing-Mlade leta, s.r.o., Bratislava, $63 \mathrm{~s}$. ISBN 978-80-10-02572-5.

9. O'CALLAGHAN FJ, SHIELL AW, OSBORNE JP, MARTYN CN (1998) Prevalence of tuberous sclerosis estimated by capture-recapture analysis. The Lancet; 351:1490.

10. RAKOWSKI SK, WINTERKORN EB, PAUL E, STEELE DJ, HALPERN EF, THIELE EA (2006) Renal manifestations of tuberous sclerosis complex: incidence, prognosis, and predictive factors. Kidney Int.;70:1777-1782.

11. ROSE VM, AU KS, POLLOM G ET AL. (1999) Germ-like mosaicism in tuberous sclerosis: how common? Am. J. Hum. Genet.; 64:986.

12. SHEPHERD CW, GOMEZ MR, LIE JT, CROWSON CS (1991) Causes of death in patients with tuberous sclerosis. Mayo Clinic Proc.;66:792-796. 